\title{
A fully parametric option in the LHCb simulation framework
}

\author{
Benedetto Gianluca Siddi ${ }^{1, *}$ \\ ${ }^{1}$ INFN Sezione di Ferrara, Via G. Saragat 1, 44122 Ferrara, ITALY
}

\begin{abstract}
Faster alternatives to a detailed, GEANT4-based simulation are being pursued within the LHCb experiment. In this context the integration of the Delphes toolkit in the LHCb simulation framework is intended to provide a fully parameterized option. Delphes is a modular software designed for generalpurpose experiments such as ATLAS and CMS to quickly propagate stable particles using a parametric approach and to provide reconstructed physical objects as output. It includes propagation inside a magnetic field and parameterized response for tracking and muon systems as well as calorimeters. Particle energies are computed by smearing the initial visible particles momenta according to detector resolution. The present contribution illustrates the current status of the Delphes toolkit in Gauss, the LHCb simulation framework. In this integration the particle transport performed by GEANT4 and subsequent mimicking of detector response and reconstruction has been replaced with a parametric response of the various detector elements. The implementation required significant changes to Delphes itself to constrain the particle transport inside the detector acceptance and to match the LHCb dipole magnetic field. The configuration of various parametrisations of resolution and efficiency is also a major aspect of the work to provide a fully functional simulation. The output of the resulting fast simulation is formatted in such a way that can be used in the $\mathrm{LHCb}$ physics analysis framework.
\end{abstract}

\section{Introduction}

Monte Carlo simulations play an important role in High Energy Physics experiments to mimic the detector response and to extract experimental conditions and performances. Moreover, they allow us to extract information and perform sanity checks that cannot be accomplished on data. Clearly, such studies requires a high level of accuracy. In the LHCb experiment [1] [2], the interaction of particles with the detector elements is simulated in detail with Geant4 [3] and requires significant amount of computing resources. In addition, for the LHCb upgrade, more complex events and larger samples will need to be simulated. The projection of available computing resources will not be enough. The simulation time is dominated by the transport of particles inside the detector $(O 95 \%$ - 99\%). For this reason increased CPU resources and also new faster Geant versions will not be sufficient and dedicated fast simulation options will be necessary. Figure 1 shows the upgrade scenario in terms of needed CPU resources. The blue line represents the required resources when $100 \%$ of the data is simulated using Geant 4 , the black line represents the pledgeable resources coming from the funding agencies.In the case where detailed simulation of the detector is performed, the amount of

\footnotetext{
*e-mail: siddi@fe.infn.it,bsiddi@cern.ch
} 


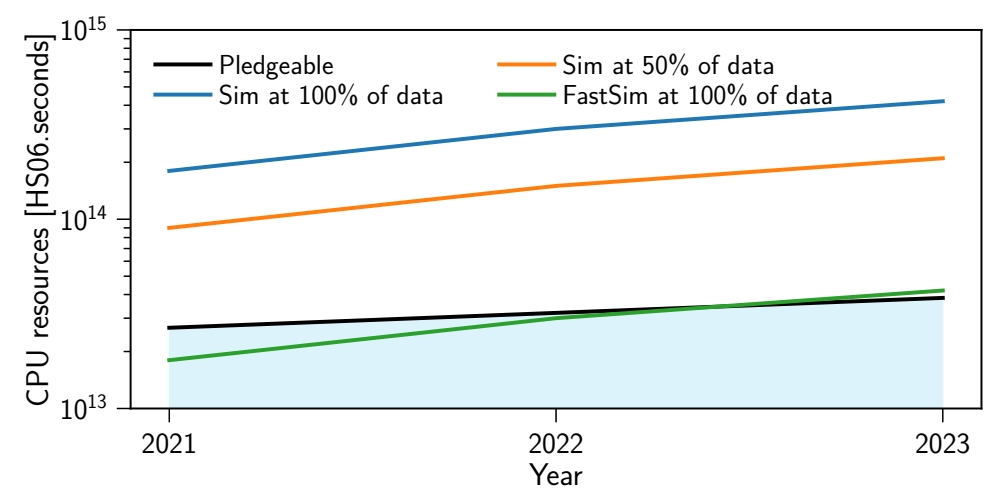

Figure 1. Possible upgrade scenario in terms of CPU resources. The black line represents the pledgeable resources coming from the funding agencies, the blue line shows the requirement in the case the $100 \%$ of the data is simulated. Orange line shows the CPU resources in the case of $50 \%$ of the data is fully simulated. The green line represent the case in which the $100 \%$ of data is simulated using fast simulation option assuming $1 / 10$ th of CPU consumption with respect to the full detailed simulation.

the necessary CPU will not be enough and other options need to be provided. The green line represent the case when the $100 \%$ of the data collected is simulated using a fast simulation option, assuming a consumption rate of $1 / 10$ th of the detailed simulation. In this scenario the available CPU resources will be sufficient. Currently, some fast simulation options are already available in the $\mathrm{LHCb}$ framework and used for production. One of them consists in the exclusion of particular sub-detectors used in the simulation, allowing a time reduction of $(O)$ $40 \%-90 \%$. Another possibility is to simulate just the decay of interest without including the rest of the event, with a time reduction of $(O) 95 \%-99 \%$, or to simulate the rest of the event once and let the signal part redecay a defined number of times. Other fast simulation options are currently under development and will be deployed in the LHCb simulation framework, Gauss [4]. In this proceeding, the status of the integration of the parametric simulation tool Delphes [5] in LHCb is given.

\section{Delphes}

Delphes [5] is a modular framework for fast parametric simulations. It is written in C++ and is available as a library. Delphes has been written originally for the ATLAS and the CMS experiments at the LHC collider and is used to parametrize not only the detector but also the reconstruction effects. Its parametric simulation includes: a tracking system embedded into a constant magnetic field, calorimeters with electromagnetic and hadronic sections, a muon system and a very forward detectors arranged along the beam-line. Delphes performs the propagation of stable particles and simulates theris interactin with the detector using a parametric approach to efficiency and resolution convolution.

\section{Delphes in LHCb}

\subsection{Output for physics analysis}

In the context of the LHCb simulation framework, Delphes will substitute the detailed simulation performed by Geant4, and will give as output only high level reconstructed objects. 
In Figure 2 a schematic view of the chain from generation to analysis in LHCb is given. Offline analyses in LHCb are performed using the DaVinci software package [6]. The DaVinci application combines basic reconstructed particles (e.g. $K, \pi, \mu$ ) and compute the relative kinematic quantities of the combined particles (e.g. $D, B, \Lambda$ ), such as invariant masses and their distance of flight. It takes as an input reconstructed basic particles and it is possible to create the combined ones and to perform candidate selection. Since the hits in the detector cannot be performed, reconstruction cannot be run and therefore output from Delphes has to directly provide objects expected by the analysis tool DaVinci. The output of Delphes is a high level reconstructed object termed "protoparticle" and containes following information on a particle:

- Links with tracks;

- At least one particle ID information;

- Link to calorimeter objects;

- Link to particle ID hypothesis objects.

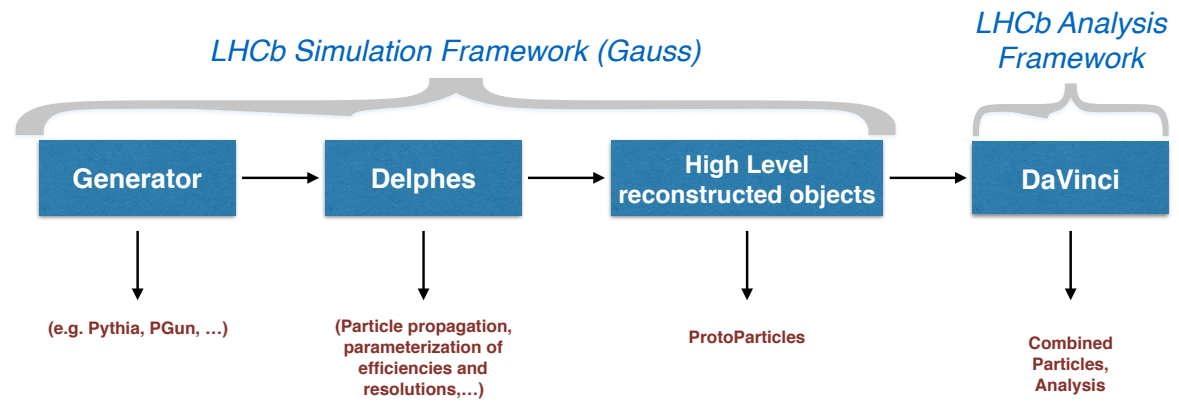

Figure 2. Schematic view of the chain from generation to analysis in $\mathrm{LHCb}$.

\subsection{Tracking parametrisation}

The original Delphes particle propagator module has been written for a solenoidal magnetic field embedded in a cylindrical acceptance and magnetic field. It has therefore been necessary to modify the functionality to match the $\mathrm{LHCb}$ acceptance. The particles are transported by applying a shift to the transverse momentum corresponding to the mean value of a simple dipole field termed $p_{T}-$ kick. Tracking resolutions and efficiencies are obtained from the LHCb detailed simulation. To include these in Delphes, two dedicated modules have been developed in order to use parametrized histograms as inputs. Efficiencies are those for reconstructed tracks in the tracker acceptance. The resolution is defined as the root mean square of differences between reconstructed Monte Carlo tracks and the Monte Carlo truth. The kinematic variables adopted to fill both histograms are the track slopes $T_{x}=p_{x} / p_{z}, T_{y}=p_{y} / p_{z}$ and the inverse of the particle momentum $1 / p$. Figure 3 shows the comparison between Delphes and detailed simulation output for the $T_{x}$ variable used for the parametrisation. A specific parametrisation for each data taking period and detector conditions is provided. Covariance error matrix associated to the track is also parametrized. This has been done with a lookup table in which each matrix element has been averaged as function of $1 . / p$. A very similar procedure is used to fill other track properties such as ghost probability [7], fit likelihood, track $\chi^{2}$ and number of degrees of freedom. All objects related to tracking are filled 


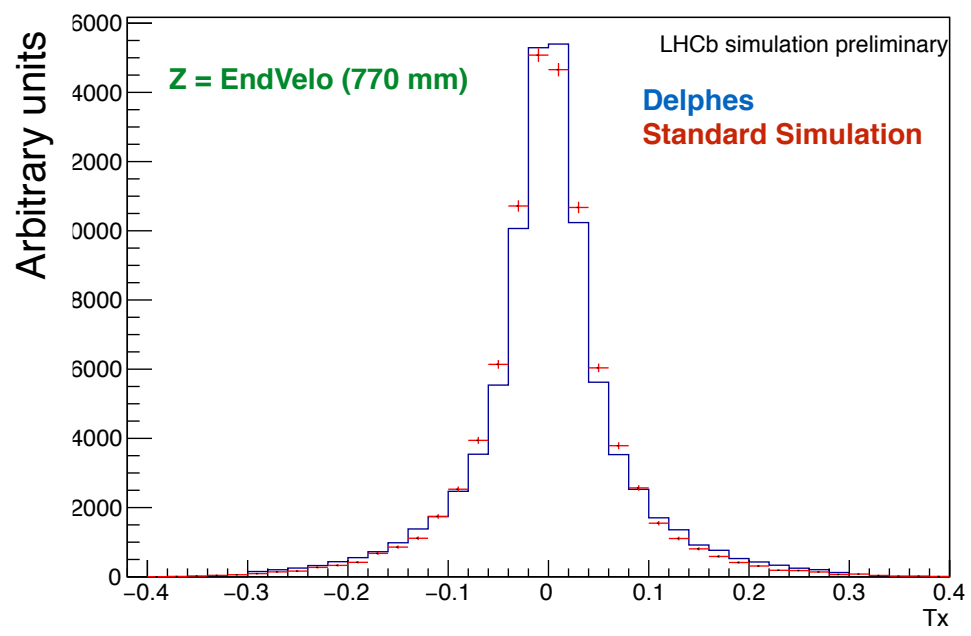

Figure 3. Track slope $T_{x}=p_{x} / p_{z}$ at the $z=770 \mathrm{~mm}$ corresponding to the end of the vertex locator of the $\mathrm{LHCb}$ detector.

and the standard $\mathrm{LHCb}$ analysis tools can be used proving the feasibility of the chain from the parametric simulation to analysis. Figure 4 shows the invariant mass of two reconstructed pions through Delphes, obtained with standard LHCb analysis framework. The next step in the

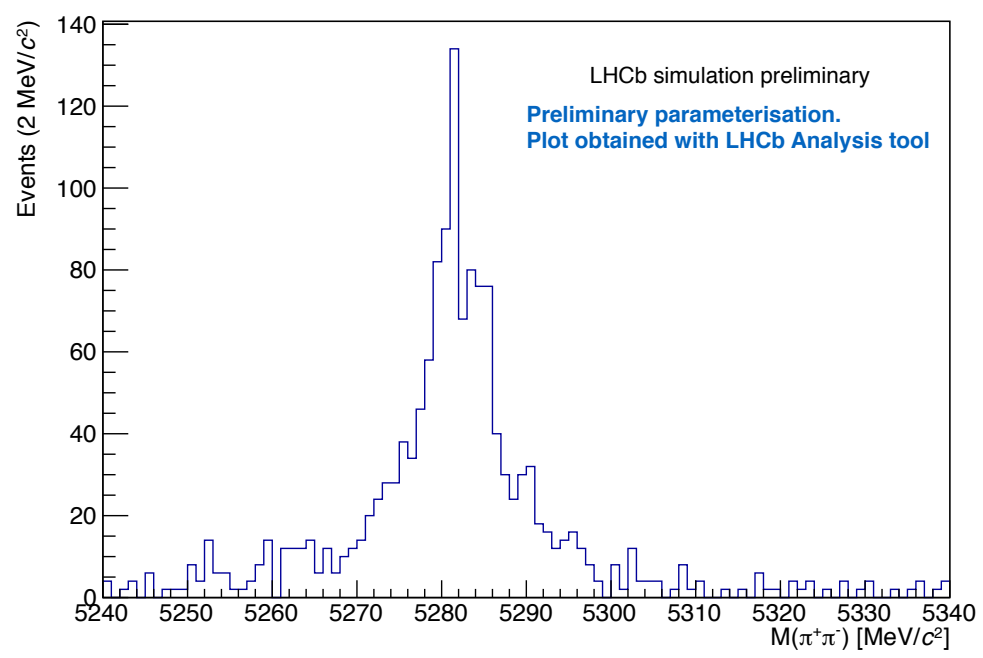

Figure 4. Invariant mass of $\pi^{+} \pi^{-}$combination obtained providing final reconstructed objects to LHCb analysis framework DaVinci.

parametrisation of the $\mathrm{LHCb}$ tracking system is to include at least one particle identification hypothesis information. 


\subsection{Electromagnetic calorimeter parametrisation}

The electromagnetic calorimeter parametrisation in Delphes has been done for neutral particles. This has been achieved using a custom geometry based on simple loops and is divided in 3 regions corresponding to the current $\mathrm{LHCb}$ calorimeter configuration. Figure 5 shows the three regions and the different granularity. The energy of each particle is obtained performing

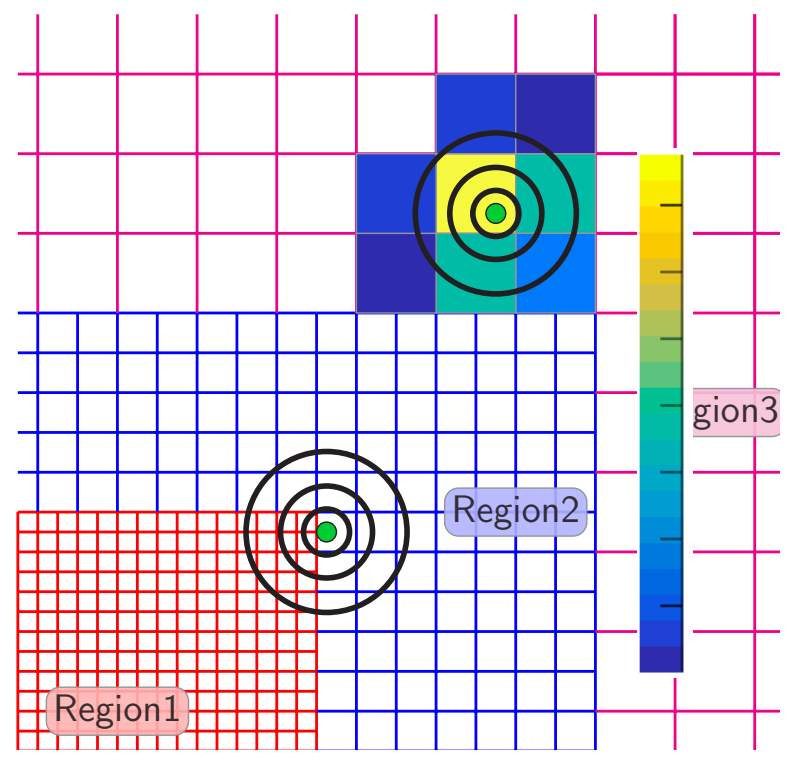

Figure 5. Schematic view of the three calorimeter regions of the LHCb detector and the different granularities.

a simple clusterization in Delphes:

- Obtain the smeared response from a Delphes module for the individual particles crossing the calorimeter surface;

- Draw circles centered in the position of the maximum energy deposit with different Molière radius, $R_{M}=1,2,3.5$, in order to get the $90 \%, 95 \%$ and $99 \%$ of the associated energy.

As an output a neutral protoparticle is made. The necessary elements needed to construct the neutral protoparticles are:

- Covariance matrix of the calotimeter cluster (Energy, $x, y$ coordinates);

- The $z$ position of the calorimeter cluster;

- Calorimeter cell IDs for every tower (built from the position);

- CaloDigits (cell ID and energy of the cell);

- Calorimeter cluster, corresponding to the collection of digits and the $z$ position of the cluster;

- At least one particle identification hypothesis.

The calorimeter parameterized response has also been tested using standard LHCb analysis tool (DaVinci). Figure 6 shows the invariant mass of two reconstruced gammas coming from a $\pi^{0}$ decay demostrating the good functionality of the calorimeter parametrisation. 


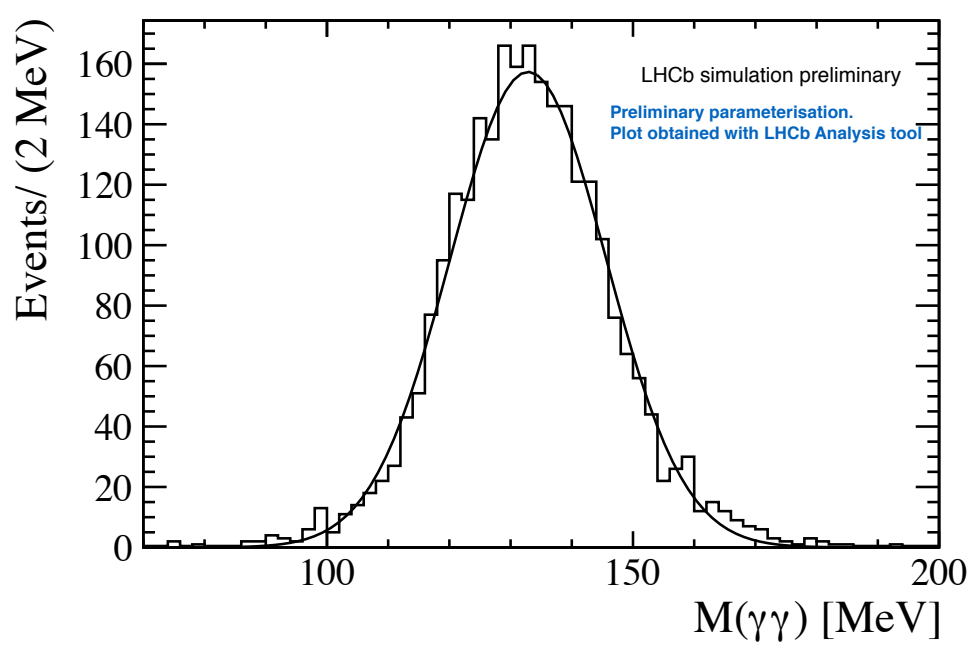

Figure 6. Schematic view of the three calorimeter regions of the LHCb detector and the different granularity.

The next steps in the calorimeter parametrisation are the generalization of the Molière radius to an ellipse and the inclusion of the parametrisation of the response of the calorimeter system for charged particles.

\section{Conclusion}

Fast simulation is crucial in LHC experiments to cope with the large amount of Monte Carlo statistics needed in the future. Delphes has been integrated within the LHCb simulation framework Gauss and extended for $\mathrm{LHCb}$ with a simple propagator for propagating particles inside the $\mathrm{LHCb}$ acceptance and a dipole magnetic field along with a parametric calorimeter response for neutral particles. Tools and algorithms have been developed in order to include tracking efficiencies and resolutions according to $\mathrm{LHCb}$ detailed simulation. A complete user analysis chain has been tested to prove that Delphes output can be used for physics analysis. Future steps will be to include the particle identification response and the calorimeter parametrisation for charged particles. The aim is to be about 100 times faster than the detailed simulation based on Geant4.

\section{References}

[1] LHCb collaboration, A. A. Alves Jr. et al., The LHCb detector at the LHC, JINST 3 (2008) S08005.

[2] LHCb collaboration, R. Aaij et al., LHCb detector performance, Int. J. Mod. Phys. A30 (2015) 1530022, arXiv:1412.6352.

[3] S. Agostinelli et al. (Geant4 collaboration), GEANT4: A Simulation toolkit, Nucl. Instrum. Meth. A506, 250 (2003);

[4] M. Clemencic et al., The LHCb Simulation Application, Gauss: Design, Evolution and Experience, J. Phys. Conf. Ser. 331, 032023 (2011) 
[5] de Favereau, J., et al., DELPHES 3: a modular framework for fast simulation of a generic collider experiment, JHEP, 2(57), 2014

[6] The DaVinci project - http://lhcb-release-area.web.cern.ch/lhcb-releasearea/doc/davinci/.

[7] Needham, M., Performance of the LHCb track reconstruction software, CERN-LHCB2007-144, LPHE-2008-01, 2007 